\title{
CLASSICAL MECHANICS WITHOUT DETERMINISM
}

\author{
Hrvoje Nikolić
}

Theoretical Physics Division

Rudjer Bošković Institute

P.O.B. 180, HR-10002 Zagreb, Croatia

E-mail: hrvoje@thphys.irb.hr

\begin{abstract}
Classical statistical particle mechanics in the configuration space can be represented by a nonlinear Schrödinger equation. Even without assuming the existence of deterministic particle trajectories, the resulting quantum-like statistical interpretation is sufficient to predict all measurable results of classical mechanics. In the classical case, the wave function that satisfies a linear equation is positive, which is the main source of the fundamental difference between classical and quantum mechanics.
\end{abstract}

Key words: classical statistical mechanics, nonlinear Schrödinger equation, particle trajectory

\section{INTRODUCTION}

It is widely accepted that quantum mechanics (QM) is a fundamentally indeterministic theory, the predictions of which are inherently probabilistic. Unfortunately, this makes QM conceptually very different from the fundamentally deterministic classical mechanics (CM). It is possible to interpret QM in a deterministic way similar to that in CM, by adopting the Bohmian interpretation 1, 2, 3, 4. In this interpretation, particles are assumed to have definite properties (positions, velocities, and continuous deterministic trajectories) even when they are not measured, which is why these properties are usually regarded as hidden variables. However, since this interpretation seems to have the same directly observable predictions as the conventional interpretation, this interpretation is usually ignored by the physics community, mainly because this interpretation is technically more complicated than the usual interpretation, without clearly leading to new measurable predictions. (The claim that there are no new measurable predictions seems to be true in nonrelativistic QM, but not necessarily in relativistic QM [5].) With such a positivistic scientific reasoning, if a determininistic theory (such as the Bohmian interpretation of QM) can be replaced with a simpler indeterministic theory (such as QM with the usual interpretation) that leads to the same measurable predictions, then it is the latter indeterministic theory that should be adopted as a more fundamental theory. However, even in classical physics, all measurements are always plagued by finite errors of measured quantities. Consequently, even in classical physics, all measurable predictions are necessarily statistical. This suggests the following interesting question: Is it possible to replace the usual deterministic approach to CM with a simpler indeterministic approach similar to QM, such that all measurable predictions of the conventional deterministic CM are preserved? By representing classical statistical mechanics by a nonlinear Schrödinger equation [6, 7, 4, 8, and by adopting a certain QM-motivated definition of "simplicity", in this paper we argue that it is possible! More specifically, we argue that this nonlinear Schrödinger equation with the appropriate purely probabilistic interpretation is 
sufficient to predict all measurable results of CM. The usual deterministic particle trajectories of $\mathrm{CM}$ play a role of unmeasured hidden variables for CM, analogous to the Bohmian hidden variables for QM. Thus, the same reasoning leading to the conclusion that QM is a fundamentally indeterministic theory may be used to argue that CM is also a fundamentally indeterministic theory.

The paper is organized as follows. In Sec. 2, we present a brief overview of QM and the corresponding Bohmian interpretation, derive the nonlinear Schrödinger equation for CM, and discuss those interpretational aspects of the latter that are not directly related to the theory of measurement. In Sec. 3, we present a general discussion of the problem of measurement in nonlinear QM and discuss the implications on the nonlinear classical Schrödinger equation. In Sec. 4. we discuss how the standard classical laws of physics (classical statistics and classical trajectories) emerge from from the quantum-like interpretation of the nonlinear classical Schrödinger equation. A discussion of our results is presented in Sec. 5.

\section{SCHRÖDINGER EQUATIONS FOR QM AND CM}

\subsection{QM and the Bohmian interpretation}

A nonrelativistic particle is described by the Schrödinger equation

$$
\left[\frac{\hat{\mathbf{p}}^{2}}{2 m}+V(\mathbf{x}, t)\right] \psi(\mathbf{x}, t)=i \hbar \partial_{t} \psi(\mathbf{x}, t),
$$

where

$$
\hat{\mathbf{p}}=-i \hbar \nabla
$$

We write $\psi$ in the polar form

$$
\psi(\mathbf{x}, t)=R(\mathbf{x}, t) e^{i S(\mathbf{x}, t) / \hbar},
$$

where $R$ and $S$ are real functions and

$$
R(\mathbf{x}, t) \geq 0
$$

The complex equation (11) is equivalent to a set of two real equations

$$
\begin{gathered}
\frac{(\nabla S)^{2}}{2 m}+V+Q=-\partial_{t} S, \\
\partial_{t} \rho+\nabla\left(\rho \frac{\nabla S}{m}\right)=0,
\end{gathered}
$$

where

$$
Q \equiv-\frac{\hbar^{2}}{2 m} \frac{\nabla^{2} R}{R}
$$

and $\rho \equiv R^{2}$. Eq. (6) is the conservation equation that provides the consistency of the interpretation of $\rho$ as the probability density. Eq. (5) is similar to the classical Hamilton-Jacobi equation, differing from it only in containing the additional $Q$-term.

The similarity of (5) with the Hamilton-Jacobi equation suggests the Bohmian interpretation consisting in the assumption that the particle has a definite trajectory $\mathbf{x}(t)$ satisfying

$$
\frac{d \mathbf{x}}{d t}=\frac{\nabla S}{m} .
$$


Eq. (8) is identical to the analogous equation in the classical Hamilton-Jacobi theory. Eqs. (8) and (6) imply that particles in a statistical ensemble with the initial probability distribution $\rho\left(\mathbf{x}, t_{0}\right)$ will be distributed according to $\rho(\mathbf{x}, t)$ at any time $t$. According to the Bohmian interpretation, the dynamics is fundamentally deterministic, while all QM uncertainties emerge from the lack of knowledge of the actual initial particle position $\mathbf{x}\left(t_{0}\right)$. The statistical predictions of the Bohmian interpretation are equivalent to those of the conventional interpretation. Since the additional assumption (8) does not lead to new measurable predictions, most physicists ignore the Bohmian interpretation and adopt the simpler, conventional interpretation that does not contain the additional equation (8).

\subsection{Classical Schrödinger equation}

Now consider a classical statistical ensemble. The probability distribution $\rho$ of particle positions satisfies the continuity equation (6). By introducing $R \equiv \sqrt{\rho}$, this equation can be written in terms of a linear operator acting on $R$ as

$$
\left[\partial_{t}+\left(\frac{\nabla S}{m}\right) \nabla+\left(\frac{\nabla^{2} S}{2 m}\right)\right] R=0 .
$$

Instead of (15), we have the classical Hamilton-Jacobi equation

$$
\frac{(\nabla S)^{2}}{2 m}+V=-\partial_{t} S
$$

By defining a new quantity $\psi$ as in (3), one finds that the classical equations (9) and (10) are equivalent to a classical analog of the Schrödinger equation [6, [7]

$$
\left[\frac{\hat{\mathbf{p}}^{2}}{2 m}+V-Q\right] \psi=i \hbar \partial_{t} \psi,
$$

where $Q$ is defined as in (7) and $\hat{\mathbf{p}}$ as in (2). In contrast to the ordinary Schrödinger equation, Eq. (11) contains an additional $Q$-term that makes the equation nonlinear in $\psi$.

The set of real equations (6) and (10) is equivalent to the complex equation (11). However, while the Planck constant $\hbar$ does not appear in (6) and (10), it does appear in the classical equation (11). Does it mean that the physical content of (11) does not depend on the value of $\hbar$ ? This is almost true, but not quite! The requirement that $R$ and $S$ should be single-valued functions does not allways guarantee that $\psi$ will also be a single-valued function. The additional requirement that $\psi$ should be single-valued may lead to physical results that depend on the value of $\hbar$. As an example, consider a particle moving circularly with the angular momentum $L_{\varphi}=m v r$, where $r$ is the radius of the circle and $v$ is the velocity. The solution of (10) is $S(\varphi, t)=L_{\varphi} \varphi-E t$, where $\varphi$ is the angle and $E$ is the energy. The requirement that $\psi$ should be single-valued means that $e^{i S(\varphi, t) / \hbar}$ should be single-valued, which reduces to the requirement that $e^{i L_{\varphi} \varphi / \hbar}$ should be single-valued. However, this requirement is nothing but the Bohr quantization condition [6]

$$
m v r=n \hbar
$$

of the so-called old quantum theory, for which it is known that correctly predicts the spectrum of the hydrogen atom. Thus, the requirement that the classical wave function $\psi$ should be singlevalued improves standard CM, in the sense that it also incorporates the old quantum theory. In fact, the Bohr-Sommerfeld postulates of the old quantum theory could have been derived from the classical Schrödinger equation as above. 
However, in CM, one does not consider Eqs. (92) and (10) as giving the complete description of classical systems. Instead, one assumes that particles have definite trajectories given by Eq. (8). On the other hand, the success of QM (without the Bohmian interpretation) suggests the following interesting question: Is it possible to recover all measurable predictions of CM just by viewing (111) as a quantum nonlinear Schrödinger equation, without assuming the additional "hidden-variable" equation (8) ? In the subsequent sections we argue that it is possible.

\section{MEASUREMENT}

\subsection{Measurement in nonlinear QM}

The problem of measurement in nonlinear QM is much more delicate than that in ordinary linear QM. For example, if one assumes that the collapse of the wave function is something that really happens during measurements, then, in contrast to linear QM, the EPR correlations can be used to transmit information instantaneously 9, 10, 11]. This suggests that the concept of wave-function collapse might not be a valid concept in nonlinear generalizations of QM. Instead, the appearance of an effective collapse in linear QM can be qualitatively explained as follows. Assume that a unit-norm solution of a linear or a nonlinear Schrödinger equation can be written as a sum

$$
\psi(\mathbf{x}, t)=\sum_{a} c_{a} \psi_{a}(\mathbf{x}, t)
$$

where $\psi_{a}$ are orthonormal functions. In the linear case, we also assume that the functions $c_{a} \psi_{a}$ themselves, as well as the functions $\psi_{a}$, are also solutions. On the other hand, it is typical of the nonlinear case that the functions $c_{a} \psi_{a}$ and $\psi_{a}$ are not solutions. Now assume that, by performing a measurement, we obtain knowledge that the actual state of the system is described by the $c_{a} \psi_{a}$ component of (13). To calculate the subsequent properties of the system at later times, it is therefore sufficient to know only the $c_{a} \psi_{a}$ component. However, since $c_{a} \psi_{a}$ is not a solution for a nonlinear case, in order to calculate the time evolution of $c_{a} \psi_{a}$, one actually has to calculate the whole solution (13), despite the fact that only one of the components is the "active" one. It is only in the linear case that $c_{a} \psi_{a}$ and $\psi_{a}$ are solutions themselves, so that $c_{a} \psi_{a}$ evolves independently of the other components and that $\psi_{a}$ evolves independently of the value of the constant $c_{a}$. In the linear case, this is why the wave function appears as if it "collapsed" to $\psi_{a}$.

An interpretation of QM that does not introduce a true collapse of the wave function is the many-worlds interpretation [12]. However, in the nonlinear case, such an interpretation leads to a communication between branches of the wave function that belong to different worlds [10]. But if there is a communication between the branches, then it is not really meaningful to say that they belong to different worlds. Thus, it is more reasonable to adopt an interpretation in which the words "many-worlds" are not taken so literally (see, e.g., Ref. [13]).

The measurement in QM can also be described in a more concrete way as follows. Assume that $\psi_{a}(\mathbf{x}, t)$ in (13) are eigenstates of some operator $\hat{A}$. Measuring $\hat{A}$ without disturbing the wave function $\psi$ can be reduced to having a correlation between the measured system with the coordinate $\mathbf{x}$ and the measuring apparatus with the coordinate $\mathbf{y}$, so that the total wave function takes the form

$$
\Psi(\mathbf{x}, \mathbf{y}, t)=\sum_{a} c_{a} \psi_{a}(\mathbf{x}, t) \phi_{a}(\mathbf{y}, t)
$$

where $\phi_{a}$ are also some orthonormal functions. A realistic measurement lasts a finite time, so such a correlation must last for a finite time. Indeed, in the linear case, if $\phi_{a}$ are chosen such that the products $\psi_{a} \phi_{a}$ are solutions (when the interaction with the measuring apparatus is 
turned on), then the wave function (14) is also a solution. On the other hand, if (13) is a solution when the interaction is turned off, it is typical of a nonlinear case that there will be no solution of the form (14) when the interaction is turned on. Instead, the solution will have a form $\Psi=\sum_{a} \tilde{c}_{a} \psi_{a} \phi_{a}$, where $\tilde{c}_{a} \neq c_{a}$. This shows that it is typically much more difficult to measure a quantity in nonlinear $Q M$ than that in ordinary linear $Q M$.

\subsection{Measurement for the classical Schrödinger equation}

After these general considerations of the problem of measurement in nonlinear QM, let us turn back to the case of nonlinear QM described by (11). Do the results of the preceding subsection imply that, in practice, it is very difficult, if not impossible, to measure anything in the quantum world described by (11)? Fortunately, the answer is no!

In the nonlinear case, a linear combination of solutions does not need to be a solution, which, as we have seen, leads to problems for quantum measurements. However, the particular form of the nonlinearity encoded in (11) with (17) reveals that, in two general cases, a linear combination of solutions is also a solution. First, if $\psi(\mathbf{x}, t)$ is a solution, then $c \psi(\mathbf{x}, t)$ is also a solution. Second, if some different solutions $\psi_{a}(\mathbf{x}, t)$ do not overlap, in the sense that

$$
\psi_{a}(\mathbf{x}, t) \psi_{a^{\prime}}(\mathbf{x}, t)=0 \text { for } a \neq a^{\prime},
$$

then a linear combination of the form of (13) is also a solution. The first property shows that the overall normalisation of the solution is arbitrary, while the second property is a manifestation of locality. Note also that the eigenstates of the momentum operator do overlap, while those of the position operator do not. This indicates that the position can be easily measured, while the momentum cannot. However, to better understand what is measurable and what is not, below we formulate the theory in a slightly different form.

Although $\psi$ does not satisfy a linear equation, there is a quantity that does satisfy a linear equation. This quantity is $R(\mathbf{x}, t)$ which satisfies the linear equation (9). Note that $R$ does not satisfy a linear equation in linear QM because there $R$ appears not only in (6) equivalent to (91), but also in (5). On the other hand, for the system described by (11), one can first solve Eq. (10) which does not contain $R$ and then insert the solution into (9) by considering $S$ as a given external function. Thus we conclude that, as far as the theory of measurement and effective collapse is concerned, the relevant "wave function" associated with (11) is not $\psi$, but $R$. As we show below, this fact, together with the positivity requirement (44), is the source of classical properties that emerge from the quantum theory described by (11). (Note, however, that it does not necessarily mean that the information contained in $S$ is lost. Indeed, one can still consider the whole complex wave function $\psi$. In this case, in the effective collapse of $\psi$, only $R$ changes, while $S$ remains the same. More on this will be said in Sec. 4.3)

Suppressing the time dependence of $R$ allows us to introduce the bra and ket notation for states $R$, in complete analogy with ordinary QM. Thus we have $R(\mathbf{x})=\langle\mathbf{x} \mid R\rangle=\langle R \mid \mathbf{x}\rangle$, where $|R\rangle$ and $\langle R|$ can be viewed as a column and row, respectively, representing the same real vector $R$ with the components $R(\mathbf{x})$. We observe that the scalar product

$$
\left\langle R_{1} \mid R_{2}\right\rangle \equiv \int d^{3} x\left\langle R_{1} \mid \mathbf{x}\right\rangle\left\langle\mathbf{x} \mid R_{2}\right\rangle \equiv \int d^{3} x R_{1}(\mathbf{x}) R_{2}(\mathbf{x})
$$

is necessarily positive (i.e., real and nonnegative). We further note that the only complete orthogonal basis $\left\{\left|R_{i}\right\rangle\right\}$ consistent with the positivity requirement $\left\langle\mathbf{x} \mid R_{i}\right\rangle \geq 0$ is the position basis $\{|\mathbf{x}\rangle\}$. Thus, the position basis is the preferred basis. The most general state consistent with the positivity requirement is

$$
|R\rangle=\int d^{3} x c(\mathbf{x})|\mathbf{x}\rangle
$$


where $c(\mathbf{x}) \geq 0$. In particular, there is no real state $R(\mathbf{x})$ that is an eigenstate of the operator (21), except for the trivial case in which the momentum eigenvalue is zero. Consequently, the state cannot "collapse" to a nontrivial eigenstate of (2), so the operator $\hat{\mathbf{p}}$ cannot be (easily) measured. This implies that, in practice, the Heisenberg uncertainty relation $\Delta x \Delta p \geq \hbar / 2$ cannot be revealed by an experiment.

Note also that despite the fact that the operator $\hat{\mathbf{p}}$ cannot be measured, the momentum can still be measured in an indirect way. In classical physics, one measures momentum by measuring two subsequent positions $\mathbf{x}_{1}, \mathbf{x}_{2}$ at times $t_{1}, t_{2}$, respectively, and defining the momentum as $\mathbf{p}=\left(\mathbf{x}_{2}-\mathbf{x}_{1}\right) / m\left(t_{2}-t_{1}\right)$. This is how the momentum can be measured indirectly in the theory described by (11).

\section{EMERGENCE OF CLASSICAL PHYSICS}

\subsection{Classical statistics}

The origin of all nonclassical (i.e., typically quantum) probabilistic phenomena of linear QM [14] (such as those related to destructive interference, EPR correlations, Bell inequalities, Hardy's paradox, etc.) can be traced back to the fact that the scalar product $\left\langle\psi_{1} \mid \psi_{2}\right\rangle$ between the probability amplitudes $\psi_{1}$ and $\psi_{2}$ does not need to be positive. Therefore, the positivity implies that there are no such nonclassical probabilistic phenomena for (11).

Similarly, for many-particle states, the particles can always be distinguished. For example, consider a symmetric 2-particle state $R(\mathbf{x}, \mathbf{y})=R_{1}(\mathbf{x}) R_{2}(\mathbf{y})+R_{2}(\mathbf{x}) R_{1}(\mathbf{y})$, where $R_{1}$ and $R_{2}$ are orthogonal. In the probability density $R^{2}$, the exchange term $2 R_{1}(\mathbf{x}) R_{2}(\mathbf{x}) R_{1}(\mathbf{y}) R_{2}(\mathbf{y})$ vanishes, which, just as in ordinary QM, implies that the two particles can be regarded as distinguishable.

Statistical physics can also be formulated in terms of density matrices. A pure state

$$
|R\rangle=\sum_{i} \sqrt{w_{i}}\left|R_{i}\right\rangle
$$

where $w_{i} \geq 0$, can be represented by a density matrix

$$
\hat{\rho}_{\text {pure }}=|R\rangle\langle R| \text {. }
$$

It should be distinguished from the associated mixed state

$$
\hat{\rho}_{\text {mix }}=\sum_{i} w_{i}\left|R_{i}\right\rangle\left\langle R_{i}\right|
$$

One finds

$$
\hat{\rho}_{\text {pure }}=\hat{\rho}_{\text {mix }}+\sum_{i \neq j} \sqrt{w_{i} w_{j}}\left|R_{i}\right\rangle\left\langle R_{j}\right| .
$$

The mixed state is a diagonal state. The pure state is not diagonal, but its diagonal part is equal to the mixed state. Is there any measurable difference between the pure state and the mixed state? Since the operator $\hat{\mathbf{p}}$ is not measurable, the most general measurable operator is of the form $\hat{A}=A(\mathbf{x})$. Since $\left|R_{i}\right\rangle$ are orthogonal, it follows that $\hat{A}$ is diagonal. Consequently, the average value of $\hat{A}$ in the mixed state is

$$
\langle\hat{A}\rangle=\operatorname{Tr}\left(\hat{\rho}_{\text {mix }} \hat{A}\right)=\operatorname{Tr}\left(\hat{\rho}_{\text {pure }} \hat{A}\right)=\int d^{3} x \rho(\mathbf{x}) A(\mathbf{x}),
$$

where $\rho(\mathbf{x})=R^{2}(\mathbf{x})$ and $R(\mathbf{x})=\sum_{i} \sqrt{w_{i}} R_{i}(\mathbf{x})$. This shows that there is no measurable difference between pure states and the associated mixed states. These states differ only in the off-diagonal 
part, but the positivity leads to the result that this part plays no measurable role. Effectively, the off-diagonal part does not appear, which is a property of classical statistical physics.

The suppression of the off-diagonal part above is similar to, but still different from, the phenomenon of decoherence in ordinary QM [15, 16]. If an experiment in ordinary linear QM is designed such that the position basis plays a role of a preferred basis, then the decoherence induced by a practically unpredictable environment leads to a classical-like statistics in which, for all practical purposes, one cannot distinguish between pure and mixed states. However, the difference between $\mathrm{QM}$ and $\mathrm{CM}$ consists it the facts that (i) in $\mathrm{CM}$ one does not need a practically unpredictable environment to supress the off-diagonal terms, (ii) in QM the influence of the environment can be supressed, so that one can observe the effects of interference, which do not have a classical analog, and (iii) in QM the position basis does not necessarily need to be the preferred one, which also allows one to perform measurements that can distinguish between $\mathrm{CM}$ and QM.

One additional comment on the difference between classical and quantum statistics is in order. Assume that some particular wave function $\psi$ satisfies both the quantum and the classical Schrödinger equation (in this case, $Q=0$ ). Does it mean that the corresponding physical state satisfies both quantum and classical statistics? The answer is no! In fact, the statistics is not defined by a single state, but by the set of all possible states to which any given state may collapse. This set is not defined by a particular solution, but by the general equation of motion - quantum or classical Schrödinger equation. (Recall that the superposition principle is crucial for the consistency of collapse, so in a classical collapse of $\psi$ only $R$ changes, while $S$ remains the same.) In this sense, in our approach one cannot say that the limit $Q \rightarrow 0$ corresponds to the classical or quantum limit of quantum or classical mechanics, respectively. (See, however, Refs. [8, 17, 18, for different approaches.)

\subsection{Classical trajectories}

It remains to see why the measurements of positions at different times are consistent with the conventional classical picture according to which the particles move as given by (8), despite the fact that we do not assume the validity of the "hidden-variable" equation (8). First, it is straightforward to see that the Ehrenfest theorem is valid for (11): The average position is

$$
\langle\mathbf{x}\rangle=\int d^{3} x \psi^{*}(\mathbf{x}, t) \mathbf{x} \psi(\mathbf{x}, t)=\int d^{3} x \rho(\mathbf{x}, t) \mathbf{x} .
$$

Using (23), (6), and (3) and integrating by parts, we find

$$
\frac{d\langle\mathbf{x}\rangle}{d t}=\int d^{3} x \rho \frac{\nabla S}{m}=\int d^{3} x \psi^{*} \frac{\hat{\mathbf{p}}}{m} \psi
$$

Similarly, using (24), (6), and (10) and integrating by parts, we obtain

$$
m \frac{d^{2}\langle\mathbf{x}\rangle}{d t^{2}}=\int d^{3} x \rho(-\nabla V)=\int d^{3} x \psi^{*}(-\nabla V) \psi .
$$

Thus, as in ordinary QM, the average position satisfies the classical equations of motion. However, the actual position may still be uncertain, so we still need to explain why particles appear as pointlike in classical mechanics, despite the fact that we do not assume (8). Of course, just as in ordinary QM, we can measure the position at a given time with arbitrary precision, which corresponds to a "collapse" of $R(\mathbf{x})$ to an arbitrarily narrow wave packet. However, in ordinary QM, even if the precision is perfect so that the wave packet is infinitesimally narrow at the given time, the wave packet will suffer dispersion, so that it will not be so narrow at later times. This 
is why particles in ordinary QM do not appear as pointlike objects with definite trajectories. On the other hand, the wave packets described by the nonlinear equation (11) behave in an entirely different way. Eq. (11) contains infinitesimally narrow soliton solutions that do not suffer dispersion. This can be seen as follows. Take an arbitrary regular solution $S$ of (10) for which $\nabla S$ and $\nabla^{2} S$ are also regular and insert this solution into (6). (Recall that one cannot do that in linear QM owing to the $R$-dependent $Q$-term in (5).) Consider a normalized Gaussian packet (not necessarily corresponding to a solution of (6) with the width $l$

$$
R_{l}(\mathbf{x}, t)=\pi^{-3 / 4} l^{-3 / 2} \exp \left[-(\mathbf{x}-\mathbf{y}(t))^{2} / 2 l^{2}\right],
$$

where $\mathbf{y}(t)$ is an as yet undetermined function. We see that $\lim _{l \rightarrow 0} \rho_{l}(\mathbf{x}, t) \equiv \lim _{l \rightarrow 0} R_{l}^{2}(\mathbf{x}, t)=$ $\delta^{3}(\mathbf{x}-\mathbf{y}(t))$. By inserting (26) into (6) and multiplying by $l$, we obtain

$$
2 \rho_{l} \frac{\mathbf{x}-\mathbf{y}}{l}\left(\frac{d \mathbf{y}}{d t}-\frac{\nabla S}{m}\right)+l \rho_{l} \frac{\nabla^{2} S}{m}=0 .
$$

Now integrate (27) over $d^{3} x$ and consider the limit $l \rightarrow 0$. Since $\nabla^{2} S$ is regular, the last term in (27) does not contribute. In the first term we introduce the new integration variable $\mathbf{z}=(\mathbf{x}-\mathbf{y}) / l$, so that equation (27) in the limit $l \rightarrow 0$ leads to

$$
\frac{2}{\pi^{3 / 2}} \int d^{3} z e^{-\mathbf{z}^{2}} \mathbf{z}\left(\frac{d \mathbf{y}}{d t}-\frac{\nabla S}{m}\right)=0 .
$$

This shows that (26) for $l \rightarrow 0$ is a solution of (9) if and only if

$$
\frac{d \mathbf{y}(t)}{d t}=\frac{\nabla S(\mathbf{y}(t), t)}{m}
$$

which finishes our proof that (11) contains infinitesimally narrow soliton solutions that do not suffer dispersion. (Do not identify (29) with (8)! While (8) describes hypothetical particle trajectories attributed to any state, (29) merely describes the motion of the crest of the wave packet (26).) Note that the derivation above may fail for linear QM because, in this case, one cannot assume (without an explicit check) that $\nabla^{2} S$ is regular for $l \rightarrow 0$, so one cannot conclude that the last term in (27) does not contribute for $l \rightarrow 0$. Of course, for some special potentials $V$ in linear QM, it is still possible that $\nabla^{2} S$ behaves such that the last term in (27) does not contribute for $l \rightarrow 0$. However, our argument above suggests that one should not expect this to be a generic feature of linear QM irrespective of $V$. Indeed, it is well known that most potentials $V$ in linear QM do not allow infinitesimally narrow non-dispersive solutions.

From the analysis above, we see that if a particle is measured to have a definite position at some time, then it will remain to have a definite position at later times and this position will change with time according to the classical equations of motion. However, in our interpretation, if the particle is not measured to have a definite position, then one is not allowed to claim that the particle has a definite position. While this interpretation contradicts the conventional interpretation of CM, it (just as in ordinary QM) does not contradict any measurable result of CM.

\subsection{Classical phase space}

The results of the preceding subsection show that there exist classical soliton states of the form

$$
\psi_{\text {sol }}(\mathbf{x}, t)=\sqrt{\delta^{3}(\mathbf{x}-\mathbf{y}(t))} e^{i S(\mathbf{x}, t) / \hbar},
$$


where $\mathbf{y}(t)$ is given by the integration of (29). Although (30) is not an eigenstate of the operator $\hat{\mathbf{p}}$, at each time $t$ one can associate with this state a definite value of the momentum

$$
\mathbf{p}(t)=\nabla S(\mathbf{y}(t), t)
$$

The value (31) is obtained when the momentum is measured indirectly, by measuring two subsequent positions at $t$ and $t+d t$. (In fact, since $\nabla \delta^{3}(\mathbf{x}-\mathbf{y}(t))$ is weakly equal to zero, perhaps it also makes sense to say that $\hat{\mathbf{p}} \psi_{\text {sol }}$ is weakly equal to $\mathbf{p} \psi_{\text {sol. }}$.) Thus, at a fixed time, one can associate both the position $\mathbf{y}$ and the momentum $\mathbf{p}$ with such a state, so, at a fixed time, we label such states as $\psi_{\mathbf{y}, \mathbf{p}}$. Such states correspond to points in the classical phase space.

Now consider a statistical ensemble of such states, $\left\{w_{\mathbf{y}, \mathbf{p}}, \psi_{\mathbf{y}, \mathbf{p}}\right\}$, where $w_{\mathbf{y}, \mathbf{p}}$ is the probability that the system is in the state $\psi_{\mathbf{y}, \mathbf{p}}$. In general, such an ensemble cannot be described by a pure state $\psi$ or $R$. Instead, such an ensemble corresponds to the most general classical statistical ensemble in the phase space and resembles the notion of mixed states in ordinary QM. The average value of an observable $O(\mathbf{x}, \mathbf{p})$ is equal to

$$
\langle O(\mathbf{x}, \mathbf{p})\rangle=\int d^{3} x \int d^{3} p w(\mathbf{x}, \mathbf{p}) O(\mathbf{x}, \mathbf{p}),
$$

where $w(\mathbf{x}, \mathbf{p}) \equiv w_{\mathbf{x}, \mathbf{p}}$ and we assume that $\mathbf{x}$ and $\mathbf{p}$ are continuous labels. As we demonstrate below, the general phase-space ensemble formulation contains the informations contained in the wave functions $\psi$ and $R$ as special cases.

As the first case, consider the case in which the initial information about the system is incoded in a pure state $\psi=R e^{i S / \hbar}$. This corresponds to a phase-space statistical ensemble in which

$$
w_{\mathbf{x}, \mathbf{p}}(t)=R^{2}(\mathbf{x}, t) \delta^{3}(\mathbf{p}-\nabla S(\mathbf{x}, t)) .
$$

If one measures $\mathbf{x}$ at $t$, then $\psi$ collapses to a wave function of the form of (30). This single measurement together with the initial information incoded in $\psi$ is sufficient to determine also the momentum p.

As the second case, consider the case in which the initial information about the system is incoded in a pure state $R$. Now this corresponds to a phase-space statistical ensemble in which $w_{\mathbf{x}, \mathbf{p}}$ does not depend on $\mathbf{p}$, i.e.,

$$
w_{\mathbf{x}, \mathbf{p}}(t)=N R^{2}(\mathbf{x}, t)
$$

where $N=1 / \int d^{3} p$ is the normalization factor. If one measures $\mathbf{x}$ at $t$, then $R$ collapses to a wave function of the form $\sqrt{\delta^{3}(\mathbf{x}-\mathbf{y}(t))}$, but the momentum $\mathbf{p}$ remains unknown. To determine $\mathbf{p}$, one must perform two measurements of $\mathbf{x}$, one at $t$ and the other at $t+d t$. Thus, two measurements are needed to determine both position and momentum incoded in the state $\psi_{\mathbf{x}, \mathbf{p}}$.

In principle, both the first and the second case are physically possible, depending on the initial information about the system that an observer knows. However, in practical experimental situations, the second case is more often than the first one.

\section{DISCUSSION}

In this paper, we have argued that all measurable properties of classical mechanics can be predicted by the quantum theory based on the nonlinear Schrödinger equation (11), without assuming the existence of particle trajectories satisfying (8). Just as in ordinary linear QM, the only question that cannot be answered without this assumption is what causes the effective wave-function collapse, i.e., what causes particles to take definite positions when $R(\mathbf{x}, t)$ is not a 
$\delta$-function? If this question is irrelevant from the predictional point of view in linear QM, then, from the same point of view, it is also irrelevant in CM represented by the nonlinear Schrödinger equation (111). Conversely, if one argues that this question is relevant in CM, so that CM cannot be considered complete without Eq. (8) that explains the effective collapse, then one can argue in the same way that linear QM also cannot be considered complete without (8). Of course, it is also consistent to adopt the conventional mixed interpretation in which $\mathrm{CM}$ is deterministic whereas QM is not, just as it is consistent to adopt a silly mixed interpretation in which QM is deterministic whereas $\mathrm{CM}$ is not. However, it seems more reasonable to adopt a symmetric interpretation in which $\mathrm{CM}$ and $\mathrm{QM}$ are either both deterministic or both indeterministic. Nevertheless, we leave the choice of the interpretation to the reader.

At the end, we also note that our results raise interesting questions on more general nonlinear generalizations of QM. In general, neither $\psi$ nor $R$ needs to satisfy a linear equation. Does it really mean that nothing can be (easily) measured in such theories? Is it the reason why such theories do not describe the real world? Or is it the reason why we do not have any observational evidence for such theories? Or does it mean that our conclusion that linearity is crucial for practical measurability should be revised? Is it the Bohmian interpretation that needs to be adopted in order to have a sound interpretation of more general nonlinear generalizations of QM? We leave the answers to these questions to the future research.

Acknowledgements. The author is grateful to anonymous referees for their objections that stimulated a more clear presentation. This work was supported by the Ministry of Science and Technology of the Republic of Croatia.

\section{References}

[1] D. Bohm, Phys. Rev. 85, 166, 180 (1952).

[2] D. Bohm and B. J. Hiley, Phys. Rev. Lett. 55, 2511 (1985).

[3] D. Bohm and B. J. Hiley, Phys. Rep. 144, 323 (1987).

[4] P. R. Holland, The Quantum Theory of Motion (Cambridge University Press, Cambridge, 1993).

[5] H. Nikolić, Found. Phys. Lett. 18, 549 (2005).

[6] R. Schiller, Phys. Rev. 125, 1100 (1962); 125, 1109 (1962).

[7] N. Rosen, Am. J. Phys. 32, 597 (1964); 33, 146 (1965).

[8] P. Ghose, Found. Phys. 32, 871 (2002).

[9] N. Gisin, Phys. Lett. A 143, 1 (1990).

[10] J. Polchinski, Phys. Rev. Lett. 66, 397 (1991).

[11] M. Czachor, Phys. Rev. A 53, 1310 (1996).

[12] B. S. DeWitt and N. Graham (Eds.), The Many-Worlds Interpretation of Quantum Mechanics (Princeton University Press, Princeton, 1973).

[13] M. Tegmark, Fortsch. Phys. 46, 855 (1998). 
[14] F. Laloë, Am. J. Phys. 69, 655 (2001).

[15] W. H. Zurek, Phys. Today 44, 36 (1991).

[16] M. Schlosshauer, Rev. Mod. Phys. 76, 1267 (2004).

[17] M. J. W. Hall, J. Phys. A 37, 7799 (2004).

[18] M. J. W. Hall and M. Reginatto, Phys. Rev. A 72, 062109 (2005). 